Article

\title{
Monitoring the Production of High Diffraction-Quality Crystals of Two Enzymes in Real Time Using In Situ Dynamic Light Scattering
}

\author{
Raphaël de Wijn ${ }^{1,+} \mathbb{C}^{\circ}$, Kévin Rollet ${ }^{1,2,+} \mathbb{C}^{\circ}$, Sylvain Engilberge ${ }^{3}$, Alastair G. McEwen ${ }^{4}$, \\ Oliver Hennig ${ }^{2}$, Heike Betat ${ }^{2}(\mathbb{D})$, Mario Mörl ${ }^{2}{ }^{(\mathbb{D}}$, François Riobé ${ }^{5}\left(\mathbb{D}\right.$, Olivier Maury ${ }^{5}{ }^{(\mathbb{D}}$, \\ Eric Girard $^{3}$ D , Philippe Bénas ${ }^{1}$, Bernard Lorber ${ }^{1}$ and Claude Sauter ${ }^{1, *}$ \\ 1 Institut de Biologie Moléculaire et Cellulaire, CNRS, UPR 9002, Architecture et Réactivité de l'ARN, \\ Université de Strasbourg, F-67084 Strasbourg, France; raphael.de.wijn@xfel.eu (R.d.W.); \\ k.rollet@ibmc-cnrs.unistra.fr (K.R.); p.benas@ibmc-cnrs.unistra.fr (P.B.); b.lorber@ibmc-cnrs.unistra.fr (B.L.) \\ 2 Institute for Biochemistry, Leipzig University, D-04103 Leipzig, German; oliver.hennig@uni-leipzig.de (O.H.); \\ heike.betat@uni-leipzig.de (H.B.); mario.moerl@uni-leipzig.de (M.M.) \\ 3 Institut de Biologie Structurale, CNRS, CEA, Université Grenoble Alpes, F-38044 Grenoble, France; \\ sylvain.engilberge@psi.ch (S.E.); eric.girard@ibs.fr (E.G.) \\ 4 Plateforme de Biologie et Génomique Structurale, \\ Institut de Génétique et de Biologie Moléculaire et Cellulaire, Université de Strasbourg, CNRS UMR 7104, \\ INSERM U 1258, F-67404 Illkirch, France; alastair@igbmc.fr \\ 5 Univ Lyon, ENS Lyon, CNRS-UMR 5182, Université Lyon 1, F-69342 Lyon, France; \\ francois.riobe@ens-lyon.fr (F.R.); olivier.maury@ens-lyon.fr (O.M.) \\ * Correspondence: c.sauter@ibmc-cnrs.unistra.fr; Tel.: +33-388-417-102 \\ + These authors contributed equally to the work.
}

Received: 25 December 2019; Accepted: 14 January 2020; Published: 23 January 2020

check for updates

\begin{abstract}
The reproducible preparation of well-diffracting crystals is a prerequisite for every structural study based on crystallography. An instrument called XtalController has recently been designed that allows the monitoring of crystallization assays using dynamic light scattering and microscopy, and integrates piezo pumps to alter the composition of the mother liquor during the experiment. We have applied this technology to study the crystallization of two enzymes, the CCA-adding enzyme of the psychrophilic bacterium Planococcus halocryophilus, and the lysozyme from hen egg white in the presence of a synthetic chemical nucleant. We were able to (i) detect early nucleation events and (ii) drive the crystallization system (through cycles of dissolution/crystallization) toward growth conditions yielding crystals with excellent diffraction properties. This technology opens a way to the rational production of samples for crystallography, ranging from nanocrystals for electron diffraction, microcrystals for serial or conventional X-ray diffraction, to larger crystals for neutron diffraction.
\end{abstract}

Keywords: enzyme; crystallization; dynamic light scattering; nucleation; nucleant; $\mathrm{Tb}-\mathrm{Xo}_{\mathrm{o}}$ crystallophore; microcrystals; nanocrystals; X-ray diffraction; XtalController

\section{Introduction}

Since its birth in the 1960s, biocrystallography has been a primary source of structural information, contributing more than $90 \%$ of 3D structures accessible in the Protein Data Bank [1] and remains a central player in structural biology, alongside NMR and CryoEM. Over the past decade, new experimental setups have been introduced that widen its applicability and transform the daily practice of crystal growers and crystallographers. The recent advent of X-ray free electron lasers (X-FEL) has enabled the serial femtosecond diffraction (SFX) analysis of micro- and nano-crystals, and offers unprecedented 
possibilities for time-resolved experiments [2-4]. At the same time, electron microscopes have been hijacked to perform micro-electron diffraction $(\mu \mathrm{ED})$, opening the way to the characterization of nanocrystals using laboratory-based instruments [5-8]. Though, like conventional ones relying on synchrotron or neutron sources, these new crystallographic approaches require crystalline material and call for the development of means facilitating the production of calibrated samples (i.e. nano-, micro-, or macrocrystals) with a size adapted to the radiation (electrons, X-rays, or neutrons) and the experimental setup.

Growing crystals of a new biomolecule (protein, DNA, RNA, and their complexes) is often a time-consuming task that involves a trial-and-error screening step to find solvent conditions generating promising crystalline or microcrystalline phases. It is followed by an optimization step to improve the quality of one or several crystalline forms and make them suitable for diffraction analysis [9]. However, before the first diffraction test, the evaluation of this two-step process mainly relies on optical microscopy observations. As a consequence, early crystal growth events, including nucleation, nano-crystal, or nano-cluster formation that directly impact the final crystallization outcome, remain hidden to the crystal grower. For this reason there is a clear need for a system enabling the preparation and the optimization of crystals under well-defined and controlled conditions, and ensuring reproducible crystalline properties and quality. The concept of such a system emerged in the nineties in the frame of a European research consortium on crystal growth (European Bio-crystallogenesis Initiative, 1998-2000) and was developed in the context of the OptiCryst European consortium (2006-2010) [10]. The current implementation called XtalController (or XC900; Xtal Concepts GmbH, Hamburg, Germany) is composed of a crystallization chamber for a single experiment with precise temperature and humidity control [11]. The composition of an initial drop (volume 5-10 $\mu$ l) of a solution of the target biomolecule can be modified by the injection of various solutions (such as water, buffer or crystallant) using two piezo injectors spraying 70 picoliter droplets (Figure 1A,A'). The sample drop can also be concentrated by evaporation of volatile chemicals (generally water) and its composition (i.e., the concentrations of components) is continuously calculated from its weight recorded to $\pm 1 \mu \mathrm{g}$ by an ultra-sensitive balance. The instrument provides diagnostic means to track the drop content along the experiment; the early occurrence of association events leading to nucleation, as well as nanocrystals can be detected in real time by dynamic light scattering (DLS) and the growth of crystals by video microscopy as soon as they reach a size exceeding a few microns. The XtalController also provides means to navigate in the phase diagram, from an undersaturated solution to a supersaturated state leading to crystal growth or precipitation. Hence, a specific phase may be stabilized or the system may be driven in the phase diagram toward another phase by varying experimental conditions in real time using the piezo injectors.

For more than three decades DLS has proven to be instrumental to study nucleation, to perform quality control of biological samples, to predict the propensity of the latter to crystallize, and, more recently, to follow their behavior in crystallization assays [12-16]. With the unique and innovative combination of piezo injectors to modify the experimental conditions and DLS to track in real time the effect of various physicochemical parameters (chemical composition, biomolecule concentration, temperature) on biomolecules in solution, the XtalController opens a wealth of possibilities for basic and applied crystallogenesis. First examples included the observation of liquid dense clusters formed during nucleation [17] and the preparation of crystals with well-defined size [18].

Here we used this technology to study the crystallization of two enzymes, the CCA-adding enzyme from the psychrophilic bacterium Planococcus halocryophilus (PhaCCA) and the hen egg-white lysozyme (HEWL). In the first case, classical vapor-diffusion assays produced numerous small crystals or precipitates. The XtalController helped better define the appropriate crystallant concentration to nucleate and grow large crystals of PhaCCA. In the second case, we used the XtalController to highlight the nucleating effect of a lanthanide complex, the crystallophore Tb-Xo4 $[19,20]$ on HEWL in the absence of crystallant. Both examples illustrate the potential of this technology in 
crystallogenesis and for the design of protocols to produce calibrated crystalline samples for a variety of crystallographic applications.
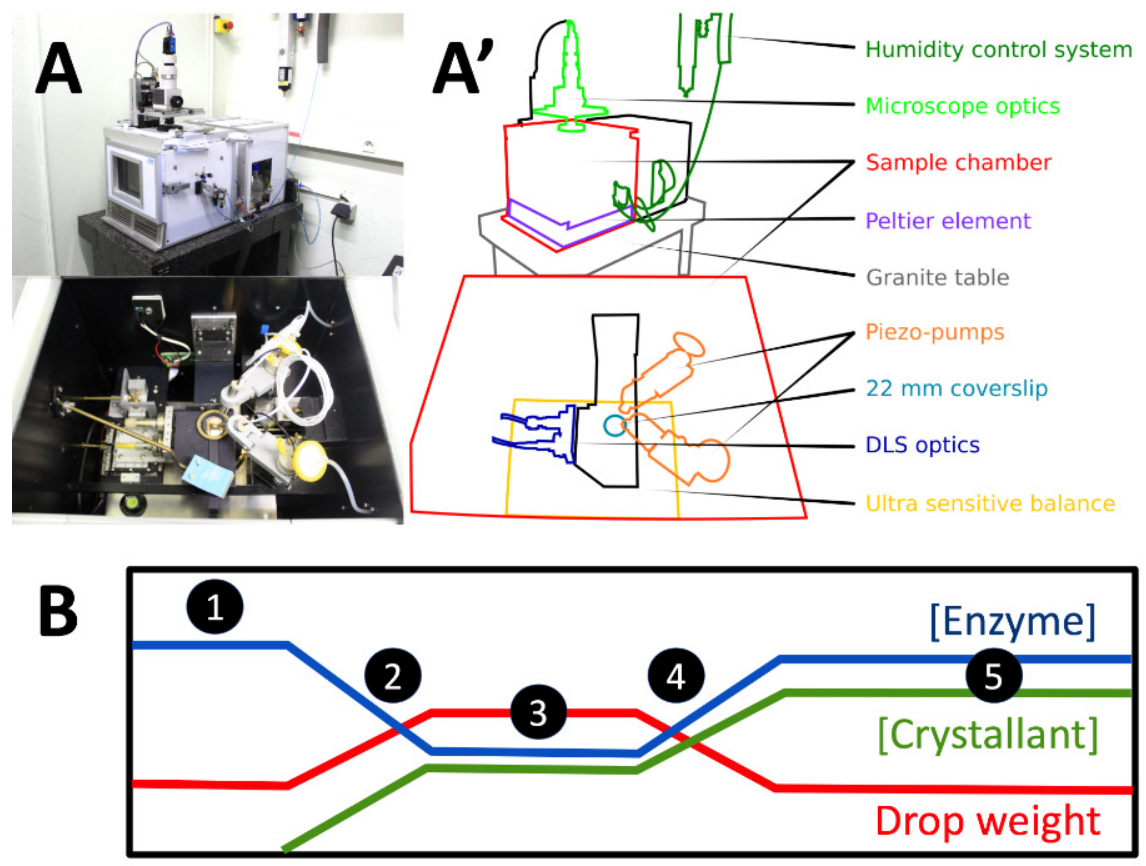

Time

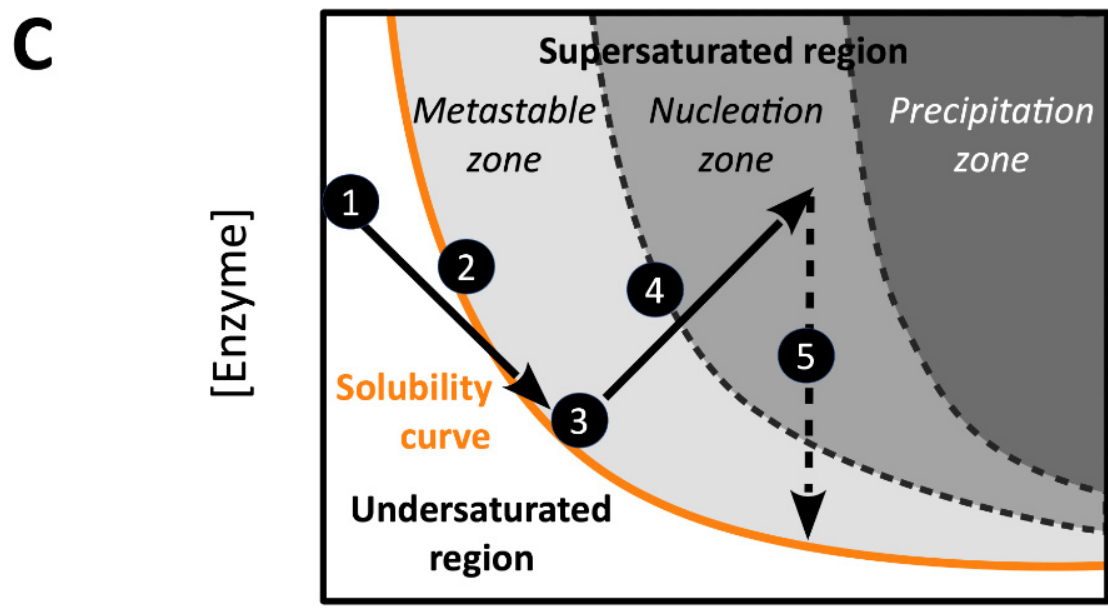

[Crystallant]

Figure 1. XtalController setup. (A) Side and top pictures of the instrument and ( $\left.\mathbf{A}^{\prime}\right)$ schematic view highlighting its major components. (B) Example of experimental schedule with curves indicating the variation of drop weight (red curve) and associated variations of enzyme and crystallant concentrations (blue and green curves, respectively). The same color code is used in all figures. (C) Corresponding trajectory in a theoretical phase diagram. Step 1: incubation of the enzyme solution at constant concentration. Step 2: addition of crystallant (increase of crystallant concentration, decrease of biomolecule concentration in the drop). Step 3: incubation, constant drop in weight (compensation of evaporation by water injection) to keep biomolecule and crystallant concentrations constant. Step 4: controlled evaporation of the drop leading to increased concentrations of biomolecule and crystallant to reach the nucleation zone. Step 5: incubation until crystals start to grow, consume part of the soluble enzyme stock and bring the system back to equilibrium on the solubility curve. 


\section{Materials and Methods}

\subsection{Chemicals and Enzyme Samples}

Chemicals used for the preparation of buffers and crystallization solutions were of highest purity grade. Solutions were filtered on $0.22 \mu \mathrm{m}$ porosity Ultrafree-MC membranes (Millipore, Molsheim, France). PhaCCA (monomer of 420 amino acids, $48 \mathrm{kDa}$ ) was produced in Escherichia coli cells, purified, concentrated to $5 \mathrm{mg} / \mathrm{mL}$, and stored at $4{ }^{\circ} \mathrm{C}$ in $50 \mathrm{mM}$ HEPES-NaOH pH 7.5, $100 \mathrm{mM} \mathrm{NaCl}$ as described previously [21]. HEWL (monomer of 129 amino acids, $14 \mathrm{kDa}$ ) was purchased from Seikagaku Corp. (Tokyo, Japan, Cat. $N^{\circ}$ 100940), Roche (Basel, Switzerland, Cat. $N^{\circ} 10153516103$ ) and Sigma-Aldrich (St. Louis, MO, USA, Fluka Cat. $\mathrm{N}^{\circ}$ 62970-5G-F). It was used without any further treatment and dissolved in milliQ water (Roche) or in $10 \mathrm{mM}$ sodium acetate pH 4.5, $40 \mathrm{mM} \mathrm{NaCl}$ (Seikagaku and Sigma) at concentrations ranging from 25 to $71 \mathrm{mg} / \mathrm{mL}$. Lysozyme stock solutions were filtered prior to concentration measurement. The crystallophore $\mathrm{Tb}-\mathrm{Xo}_{0} 4$ used as nucleant for HEWL was synthesized and purified as described [19]. It was dissolved in water to prepare a $100 \mathrm{mM}$ stock solution.

\subsection{Crystallization in the XtalController}

The humidity and temperature of the crystallization chamber of the instrument were set to $99.5 \%$ and $20.0^{\circ} \mathrm{C}$, respectively, one hour before starting an experiment to ensure the stability of experimental conditions. A drop of $10 \mu \mathrm{l}$ of enzyme stock solution was deposited on a siliconized glass coverslip (Ø $22 \mathrm{~mm}$ ) placed on the balance. One pump was loaded with the appropriate crystallant solution and the second with pure water. The crystallization chamber was closed and the protocol started with a $5 \mathrm{~min}$ step to monitor drop evaporation and compensate the loss of weight by injection of water to keep the drop weight constant. Several steps of crystallant addition, drop evaporation or dilution were scheduled to explore the phase diagram. The shooting frequency of the pumps was adjusted to vary the slope of concentration variations. DLS measurements and drop image capture were scheduled at regular time intervals (e.g., every 5 to $30 \mathrm{~min}$ ) to follow nucleation, aggregation, or crystal growth events. At the end of the experiment, the coverslip was transferred onto a 24-well Linbro plate (HR3-110, Hampton Research, Aliso Viejo, CA, USA) for incubation.

\subsection{Standard DLS Measurements}

In parallel to DLS measurements performed inside the XtalController, a benchtop Nanostar light scattering instrument (Wyatt Technology, Inc., Santa Barbara, CA, USA) was used to record the effect of Tb-Xo4 on lysozyme. Total of $10 \mu \mathrm{l}$ of Sigma lysozyme $(71 \mathrm{mg} / \mathrm{mL})$ were transferred into a quartz cell for DLS measurements at $20^{\circ} \mathrm{C}$. The drop was covered with $10 \mu \mathrm{l}$ paraffin oil and the cuvette was sealed with Parafilm ${ }^{\mathrm{TM}}$ foil to prevent evaporation. Subsequently, $1 \mu \mathrm{l}$ of a $100 \mathrm{mM}$ Tb-Xo4 stock solution in $10 \mathrm{mM}$ sodium acetate $\mathrm{pH} 4.5$ was added. In control experiments the Tb-Xo4 solution was replaced by buffer. Data were corrected for solvent viscosity and refractive index.

\subsection{Crystal Analysis}

Crystals of PhaCCA were analyzed in cryogenic conditions at FIP/BM30A beamline (ESRF, Grenoble, France) using an ADSC Quantum 315r detector. A crystal was soaked for a few seconds in the mother liquor supplemented with $20 \%(\mathrm{w} / \mathrm{v})$ glycerol, mounted in a cryoloop and flash-frozen in liquid nitrogen. 240 images were collected with a rotation of $0.5^{\circ}$ and an exposure time of $1 \mathrm{~s} \mathrm{per}$ frame, yielding a dataset at a resolution of $2.28 \AA$. Crystals of HEWL were analyzed at ambient temperature using a Rigaku FR-X diffractometer at the FRISBI platform (IGBMC, Illkirch, France) with an EIGER $\mathrm{R} 4 \mathrm{M}$ detector (DECTRIS) with a $2 \theta$ offset of $10^{\circ}$. Several crystals grown with Roche, Sigma, and Seikagaku lysozymes were tested and diffracted to up to $1.5 \AA$ resolution but showed rapid decay because of radiation damage at ambient temperature. The exposure time and rotation speed were adapted accordingly to collect a full dataset from a crystal of Seikagaku lysozyme plunged in viscous Parabar 10312 (Hampton Research), mounted in a cryoloop and protected from dehydration using the 
MicroRT room temperature kit from MiTeGen. 720 images were collected with a rotation of $0.25^{\circ}$ and an exposure time of $2 \mathrm{~s}$ per frame. Data were processed with the XDS package [22].

\subsection{Structure Determination}

The structures of PhaCCA and HEWL were refined in PHENIX [23] using PDB entries 6QY6 and 6F2I (cleared of solvent molecules and ligands), respectively, as starting models for initial rigid body adjustment. Several rounds of refinement and manual inspection in COOT [24] were performed until convergence of $\mathrm{R}_{\text {free }}$ (calculated using 5\% of reflections). The model of PhaCCA was refined using one TLS group and includes 365 residues (the $\mathrm{N}$-terminal expression tag and the flexible loop encompassing residues 83-94 are not visible), two phosphate ions, two acetate ions, and three glycerol molecules present in the mother liquor and the cryoprotection solution. The model of HEWL was refined using anisotropic atomic displacement parameters (ADPs) and includes the full enzyme sequence, one sodium, two chloride ions, a full $\mathrm{Tb}$-Xo4 complex and two additional $\mathrm{Tb}^{3+}$ positions clearly identified in the anomalous density map but for which the ligand was not observed because of low occupancy.

\section{Results and Discussion}

\subsection{Triggering the Growth of Large Crystals of PhaCCA}

We recently reported the crystallization of a new tRNA nucleotidyltransferase or CCA-adding enzyme from the cold-adapted bacterium P. halocryophilus living in the permafrost [21]. During the process of optimization we observed that the protein had a tendency to produce either clear drops or drops full of small crystals or light precipitate, indicating a narrow nucleation zone. To increase the reproducibility of crystallization, microseeding was systematically used, both in vapor-diffusion or in counter-diffusion assays [21,25]. In the present work the goal is to exploit the XtalController technology to determine appropriate crystallant concentration sufficient for the nucleation of a minimal number of crystals.

Figure 2 shows a typical crystallization assay with PhaCCA. The experiment starts with a drop of enzyme at $5 \mathrm{mg} / \mathrm{mL}$ in its storage buffer. The crystallant (stock solution: $1 \mathrm{M}$ diammonium phosphate, $0.1 \mathrm{M}$ ammonium acetate $\mathrm{pH} 4.5$ ) is gradually added into the enzyme solution using a piezo pump. The DLS signal clearly indicates the start of protein association around $0.2 \mathrm{M}$ diammonium phosphate with the appearance of objects larger than the initial PhaCCA monomer and increasing in size. This phenomenon is amplified during the step of drop evaporation when both the concentration of crystallant and enzyme simultaneously increase, hence increasing the supersaturation of the enzyme solution. The monomeric form of PhaCCA remains present throughout the experiment, in equilibrium with nano/microcrystalline objects.

After $48 \mathrm{~h}$, the monitoring was stopped and the cover slip was transferred from the instrument to a classical 24-well Linbro plate to be stored in equilibrium with a reservoir containing a crystallant solution at the same concentration as that reached at the completion of the protocol (i.e., $1 \mathrm{M}$ diammonium phosphate, $0.1 \mathrm{M}$ ammonium acetate $\mathrm{pH}$ 4.5). Small crystals that were already visible after $28 \mathrm{~h}$ in the periphery of the drop, grew slowly to a useful size (100-200 $\mu \mathrm{m})$ for X-ray diffraction (see Section 3.3) over a period of several weeks.

In an attempt to increase the size of PhaCCA crystals, protocols including several cycles of drop concentration/dilution were tested. The experimental idea was to trigger protein interaction, then redissolve partially the population of nuclei to select a few for growth in the next drop concentration step. Figure 3 illustrates this strategy and displays the resulting crystals. The largest reached a size of $0.5 \mathrm{~mm}$ after 11 days of incubation. This strategy may be exploited in the future to grow large crystals for neutron diffraction. A similar concept was applied using temperature variation coupled with dialysis to adjust the supersaturation and reduce the number of nuclei to promote the growth of large mm-sized crystals [26]. The great advantage of the XtalController is the possibility to monitor the fluctuation of particle populations in real time. The DLS signal shows that the monomer of PhaCCA 
is converted into larger objects during concentration steps while the drop remains optically clear. If the process is too fast and evolves toward precipitation, the whole system can be driven back to lower concentrations favoring the dissolution of precipitates and the re-solubilization of monomers. A series of cycles of decreasing concentration variation allows a slow and controlled convergence to the target condition, similar to that used in classical vapor diffusion assays but resulting in many fewer nucleation events and, thus, in larger crystals.
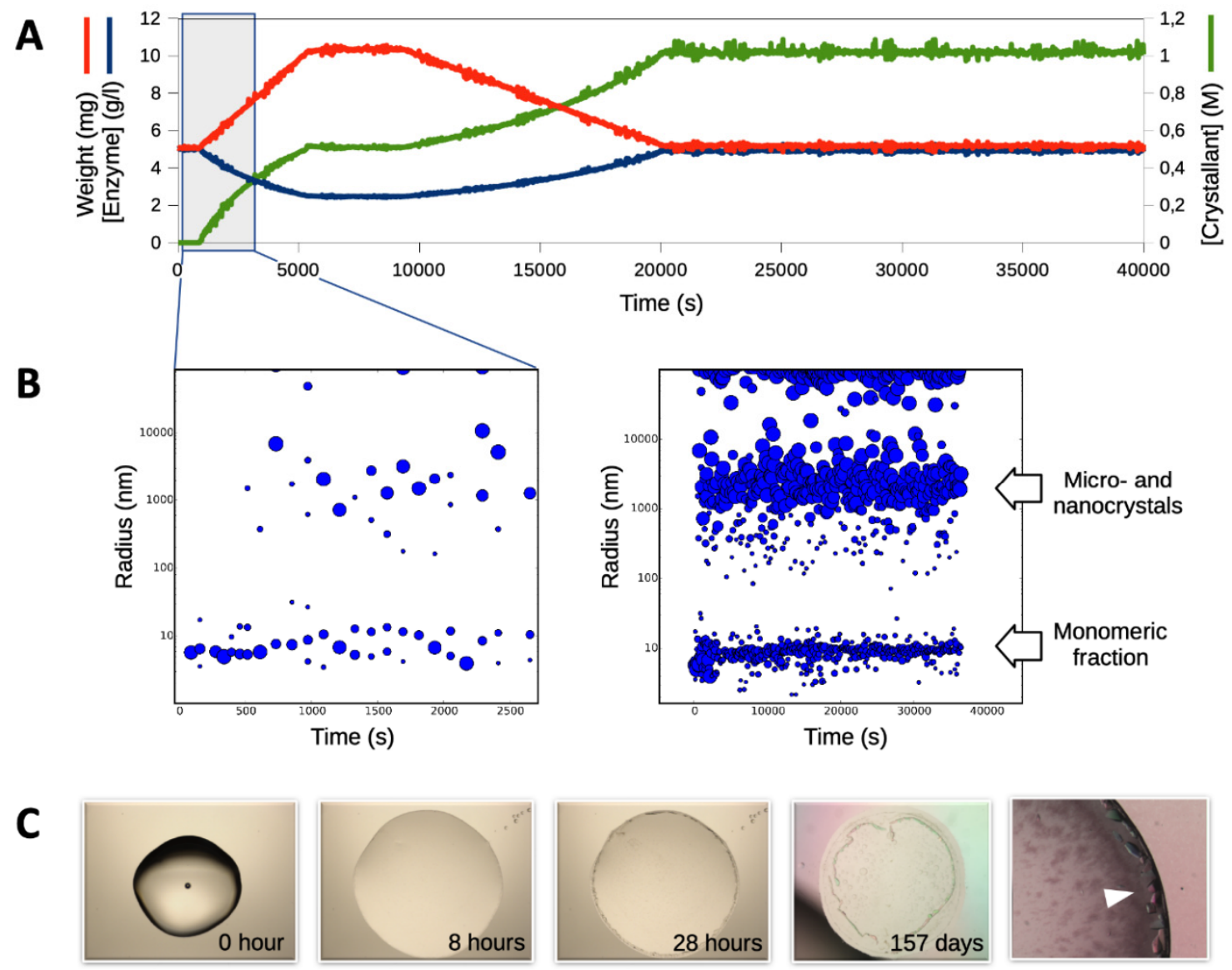

Figure 2. Crystallization of PhaCCA enzyme in the XtalController. Panels (A) and (B) show the experimental records over the first $40,000 \mathrm{sec}(\sim 11 \mathrm{~h})$. No visible change occurred between $t=11$ and $48 \mathrm{~h}$, i.e., when the experiment was transferred to a Linbro plate. (A) Experimental parameters are recorded and curves display the variation of drop weight and corresponding variation of enzyme and crystallant concentrations over time. (B) The evolution of particle size in solution is monitored by dynamic light scattering (DLS) over time. At each time point, different populations detected in solution are represented as blue dots. The center of the dot indicates the hydrodynamic radius $R_{h}$ of the population and the surface its relative contribution to the overall scattered signal. The DLS distribution on the left highlights early events (blue window in A) and shows that the enzyme starts to react at concentrations of crystallant as low as $0.2 \mathrm{M}$. Particle size distribution on the right corresponds to the monitoring of the complete experiment. The signal of monomeric PhaCCA $\left(R_{h}=4 \mathrm{~nm}\right)$ decreases while larger objects, likely nano-crystals or nano-clusters, appear upon crystallant addition. (C) A selection of drop micrographs taken along the experiment shows the appearance of small objects after $28 \mathrm{~h}$, leading to useful samples for diffraction analysis within several weeks of incubation over a reservoir in a Linbro plate. The close-up view on the right-hand side shows typical PhaCCA crystals (white arrow) used for data collection (Table 1). 

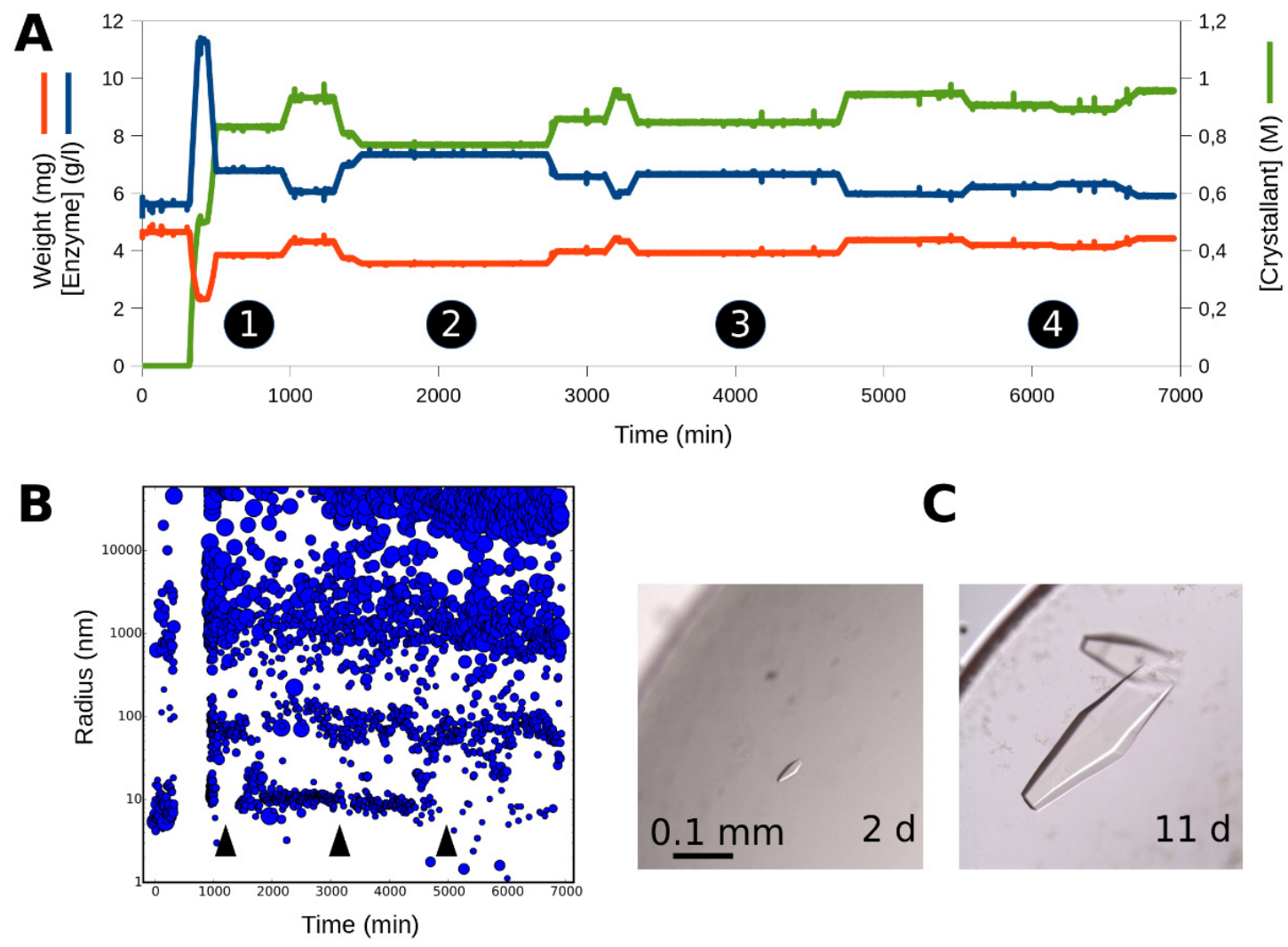

Figure 3. Production of large PhaCCA crystals. (A) The crystallant was injected in the sample drop following the same protocol as in Figure 2. Four cycles (see numbers) of drop concentration/dilution were performed. (B) The DLS monitoring shows that each time the drop is concentrated, the signal for the population of monomeric PhaCCA decreases (see arrows) in favor of larger particles. At the end of this protocol, the clear drop was transferred from the XtalController to a Linbro plate for incubation.

(C) First crystals appeared after 2 days and the biggest reached a size $0.5 \mathrm{~mm}$ in 11 days.

\subsection{Tracking the Nucleant Effect of the Crystallophore Tb-Xo4on HEWL}

A terbium complex, called crystallophore $\mathrm{N}^{\circ} 1$ or $\mathrm{Tb}-\mathrm{Xo}_{\mathrm{o}}$, with phasing and nucleating properties was recently described $[19,20,27]$. When preparing lysozyme solutions in pure milliQ water including $10 \mathrm{mM} \mathrm{Tb}-X_{\mathrm{o}} 4$ for screening experiments, we observed that the addition of the compound led to the formation of a pellet composed of single lysozyme crystals at the bottom of Eppendorf tubes after 20 to 30 days in the cold room. To confirm the ability of the nucleant to promote lysozyme self-association in conditions containing very low salt concentration, the XtalController was used to monitor the behavior of the enzyme in solution upon injection of $10 \mathrm{mM} \mathrm{Tb}$-Xo4 dissolved in water. The control experiment consisted in injecting an equivalent volume of pure water. After incubating $10 \mu \mathrm{l}$ of a fresh HEWL solution $(25 \mathrm{mg} / \mathrm{mL})$ at $20^{\circ} \mathrm{C}$ to check its stability by DLS (Figure 4), the crystallization chamber was opened for a few seconds to inject $1 \mu \mathrm{l}$ of a $100 \mathrm{mM}$ Tb-Xo4 solution (or water) directly into the drop using a $1 \mu \mathrm{l}$ Hamilton syringe. Accordingly, the drop weight increased to $11 \mathrm{mg}$ and rapidly went back to $10 \mathrm{mg}$ upon water evaporation controlled by the instrument scheduler. The drop was incubated for hours and DLS measurements were recorded before its transfer to a Linbro plate for long-term storage.

Figure 4 shows that the addition of $\mathrm{Tb}-\mathrm{Xo}_{\mathrm{o}}$ triggered the instantaneous formation of particles of larger size and this phenomenon of association, which was not observed upon water addition, led to the growth of large crystals after weeks of incubation. Tb-Xo4 decreases the solubility of HEWL and promotes interactions between enzyme particles: the monomer seems to be converted in a slightly larger entity (Figure 5), possibly bridged by the nucleant (Figure $6 \mathrm{~B}, \mathrm{C}$ ) and large assemblies grow up to a size of $1 \mu \mathrm{m}$. Again, the XtalController was instrumental to highlight a situation leading 
to nucleation in a drop that remained clear for hours or days in videomicroscopy. Indeed, when a supersaturated state has been created, the system is thermodynamically set for either precipitation or nucleation. If nuclei are favored over precipitates, then crystal growth is a matter of time (i.e., kinetics). The "activated" drop is then simply transferred from the crystallization chamber to a Linbro plate for incubation until crystals grow.

A
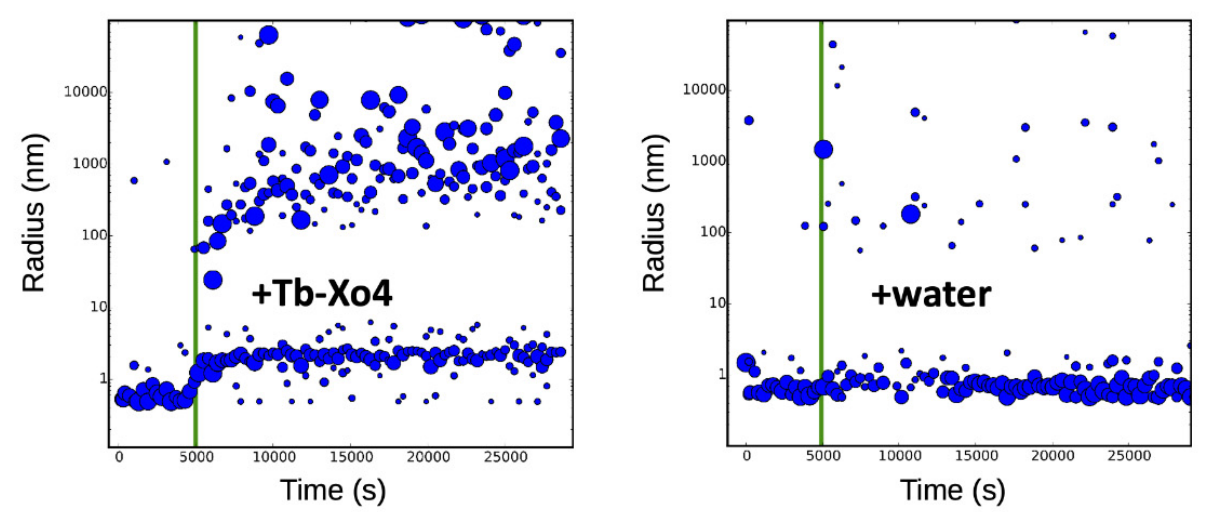

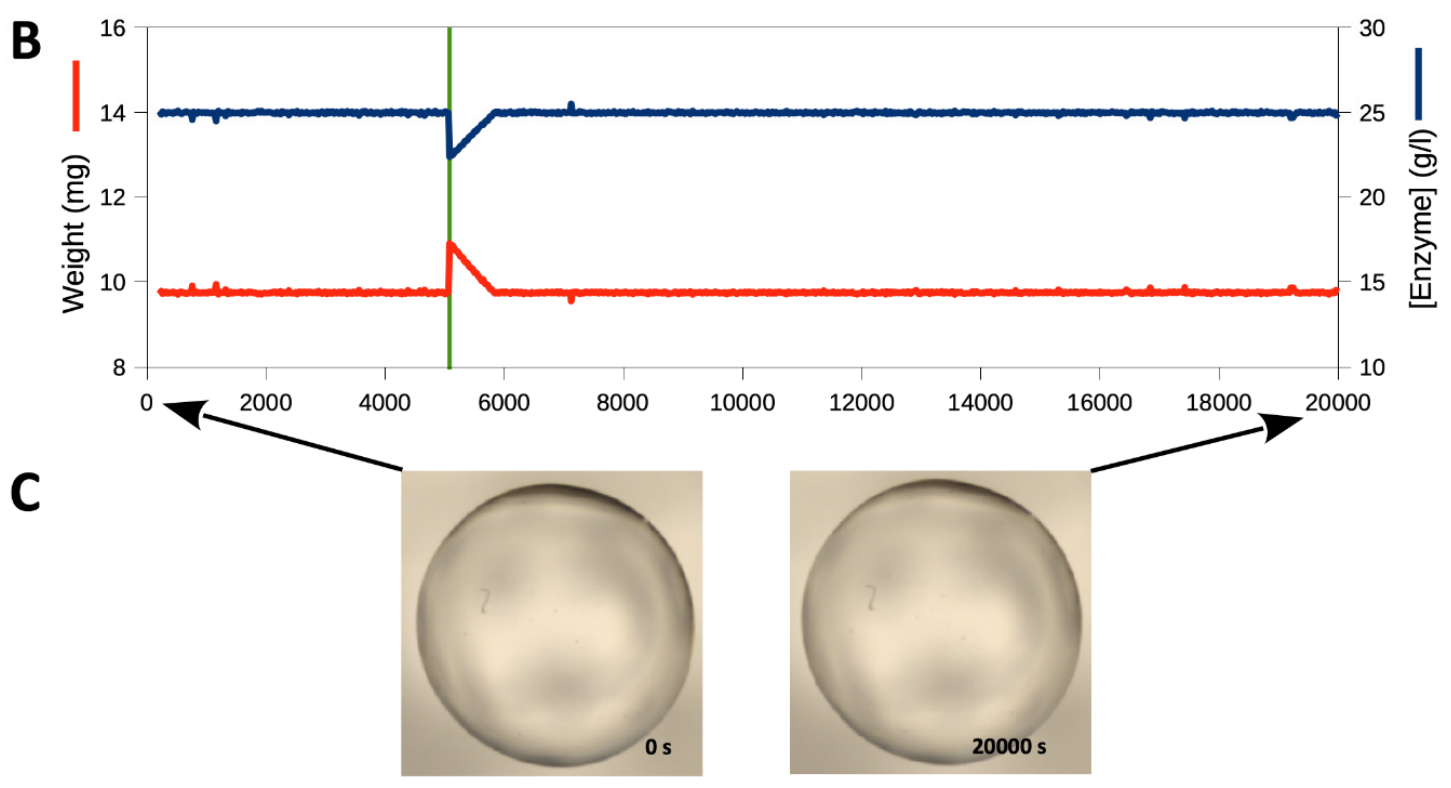

Figure 4. Monitoring the nucleant effect of Tb-Xo4 on HEWL. In this simple experiment, a Roche lysozyme solution $(25 \mathrm{mg} / \mathrm{mL}$ in water) was incubated in the XtalController. (A) Upon injection of $1 \mu \mathrm{l}$ of nucleant (100 mM Tb-Xo4 solution), symbolized by the green line and highlighted by a $1 \mathrm{mg}$ jump of the weight (B), the enzyme immediately reacted and formed larger objects that grew up to size of $\sim 1 \mu \mathrm{m}$ (A, left). The drop was then maintained at a constant weight as illustrated by weight and concentration curves (B). Records (A) and (B) are displayed for $\mathrm{t}=0$ to $20,000 \mathrm{sec}(\sim 5.6 \mathrm{~h})$. No visible change was observed afterwards and, as the drop remained clear $(\mathbf{C})$, it was transferred after $8 \mathrm{~h}$ to a Linbro plate for incubation. The control injection of $1 \mu \mathrm{l}$ of water does not change the DLS signal (A, right).

We further tested the nucleating property of $\mathrm{Tb}-\mathrm{Xo}_{\mathrm{O}} 4$ on the crystallization of HEWL from other suppliers (Sigma and Seikagaku) to check for potential enzyme preparation and batch effect. Following the protocol described in Figure 4, the concentration of enzyme was increased to 50 and $71 \mathrm{mg} / \mathrm{mL}$ to promote the growth of larger crystals. As for the Roche HEWL, the addition of Tb-Xo4 triggered the rapid formation (i.e., within 15 to $30 \mathrm{~min}$ ) of populations of particles with radii ranging from 100 to $1000 \mathrm{~nm}$ in the XtalController (Figure 5A). The same phenomenon was reproduced in the quartz cuvette of a Nanostar DLS instrument using the same lysozyme batch. Although they contributed significantly to the scattering signal, the percentage in mass of the larger populations was always low, 
in the range of a few percent (Figure 5B). Drops prepared with Sigma and Seikagaku lysozyme batches in the XtalController and incubated for several months against a buffer reservoir produced crystals of several hundreds of $\mu \mathrm{m}$ suitable for crystallographic analyses (Figure 5C).

Table 1. Statistics of crystal analysis and structure refinement.

\begin{tabular}{|c|c|c|}
\hline Enzyme & PhaCCA & HEWL \\
\hline \multicolumn{3}{|l|}{ Data collection } \\
\hline X-ray source & FIP/BM30A - ESRF & Rigaku Fr-X - IGBMC \\
\hline Wavelength $(\AA)$ & 0.9799 & 1.5418 \\
\hline Detector & ADSC Q315r & EIGER R 4M \\
\hline Temperature (K) & 100 & 293 \\
\hline Space group & $P_{4}{ }_{3}{ }_{1} 2$ & $P_{4}{ }_{3}{ }_{1} 2$ \\
\hline Cell parameters $(\AA)$ & $70.53,70.53,291.48$ & $78.81,78.81,38.33$ \\
\hline Mosaicity $\left({ }^{\circ}\right)$ & 0.31 & 0.12 \\
\hline Solvent content & 67.4 & 40.8 \\
\hline Resolution range $(\AA)$ & $47-2.28(2.42-2.28)$ & $35-1.51(1.60-1.51)$ \\
\hline Number of reflections & $237664(37915)$ & $164648(14601)$ \\
\hline Number of unique reflections & $34470(5385)$ & 19535 (3017) \\
\hline Multiplicity & $6.9(7.0)$ & $8.4(4.8)$ \\
\hline Completeness (\%) & $99.5(98.5)$ & $99.2(96.5)$ \\
\hline Mean I/sigma(I) & $15.5(1.5)$ & $29.7(1.9)$ \\
\hline$R_{\text {meas }}(\%)^{1}$ & $8.6(126)$ & $3.6(72.6)$ \\
\hline$R_{\text {pim }}(\%)^{2}$ & $3.4(44.8)$ & $1.1(31.7)$ \\
\hline SigAno & - & $2.1(0.6)$ \\
\hline $\mathrm{CC}_{1 / 2}$ & $99.9(76.0)$ & $100(78.1)$ \\
\hline \multicolumn{3}{|l|}{ Structure refinement } \\
\hline$R_{\text {work }}, R_{\text {free }}$ & $0.213,0.257$ & $0.141,0.175$ \\
\hline $\begin{array}{l}\text { Number of non-H atoms } \\
\text { enzyme, ligands, solvent }\end{array}$ & $2989,36,105$ & $1001,35,54$ \\
\hline RMSD on bonds $(\AA)$ and angles $\left(^{\circ}\right)$ & $0.009,0.96$ & $0.004,0.69$ \\
\hline $\begin{array}{l}\text { Average ADPs }\left(\AA^{2}\right) \\
\text { overall, enzyme, ligands, solvent }\end{array}$ & $51.0,50.8,62.9,51.6$ & $30.1,28.7,51.7,41.9$ \\
\hline $\begin{array}{l}\text { Ramachandran plot: \% of residues in } \\
\text { favored, allowed, unfavored regions }\end{array}$ & $96.4,3.3,0.3$ & $99.2,0.8,0$ \\
\hline PDBid & $6 \mathrm{TVZ}$ & $6 \mathrm{TVY}$ \\
\hline
\end{tabular}



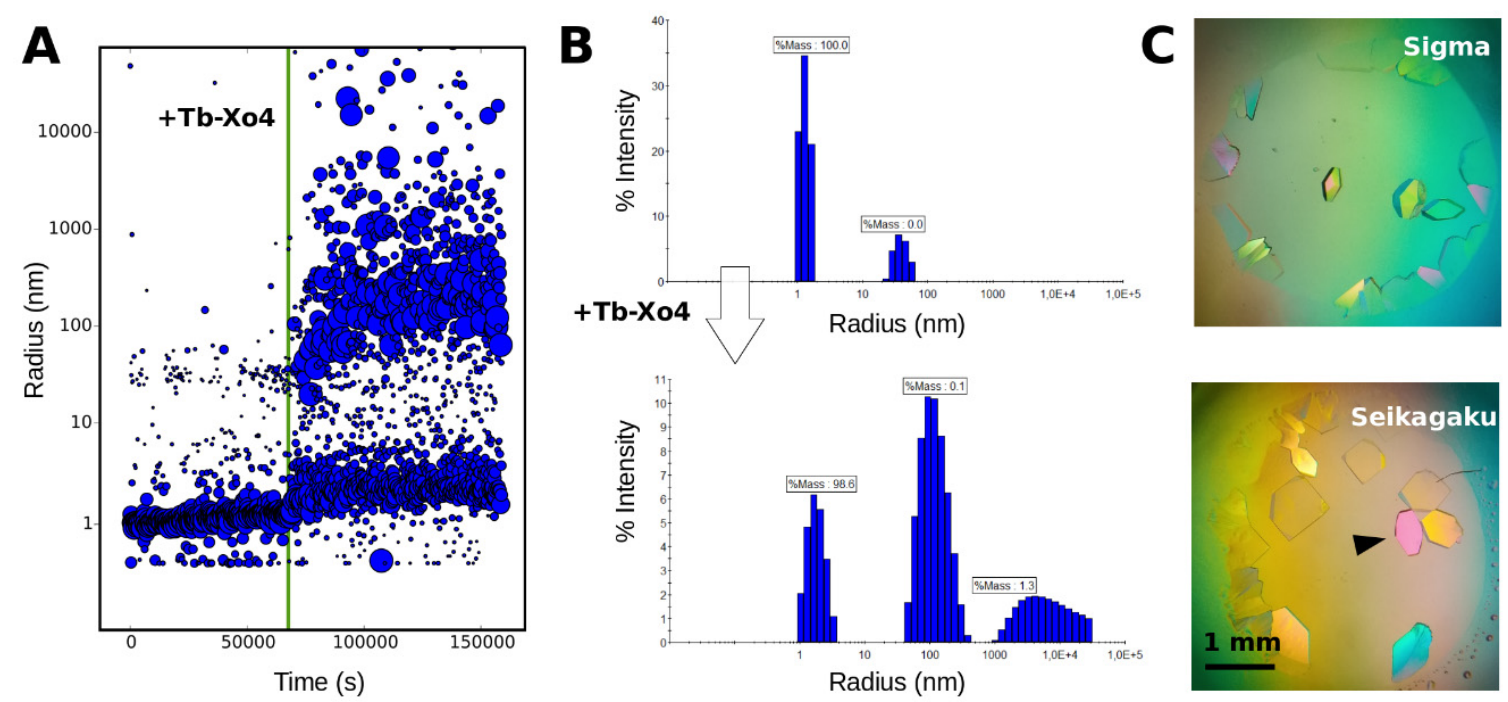

Figure 5. Production of Tb-Xo4-HEWL crystals for structural analyses. The experimental procedure described in Figure 4 was applied to Sigma and Seikagaku lysozyme samples at a higher concentration (50 and $71 \mathrm{mg} / \mathrm{mL}$, respectively) to promote the growth of larger crystals. (A) the DLS monitoring of Sigma lysozyme in the XtalController upon Tb-Xo4 injection (green line) shows same features as in Figure 4A, yet amplified by the higher enzyme concentration. (B) The phenomenon was followed on the same batch in a Nanostar DLS instrument. The top distribution detects almost exclusively the monomeric lysozyme before the injection, whereas populations with a $R_{h}$ of $\sim 100 \mathrm{~nm}$ and $>1 \mu \mathrm{m}$ appear in the bottom distribution already $2.5 \mathrm{~min}$ after $\mathrm{Tb}-\mathrm{Xo}_{\mathrm{O}}$ injection. Crystallization assays performed with both batches were transferred from the XtalController to a Linbro plate for incubation. (C) Images showing resulting crystals after 9 months before harvesting for data collection. The crystal marked by an arrow was used to collect the dataset presented in Table 1.

\subsection{Characterization of Crystals Grown in the XtalController}

The quality of PhaCCA and HEWL crystals grown using the XtalController was assessed by X-ray diffraction. Crystals of PhaCCA were analyzed under cryogenic conditions at the FIP-BM30A synchrotron beamline (ESRF, Grenoble, France). Table 1 shows excellent statistics for such a crystal leading to a complete diffraction dataset at $2.28 \AA$ resolution. The signal extended isotropically up to $1.8 \AA$ (not shown) as for our best data collected on a much stronger beamline installed on an insertion device (PROXIMA2A, SOLEIL, Saint-Aubin, France). Here, data were truncated because of the presence of two diffraction rings at resolutions of about 1.9 and $2.15 \AA$. They were probably due to suboptimal cryocooling of the solvent and significantly altered the statistics in the high resolution shell $(<2.2 \AA)$. However, the resulting electron density maps are extremely clear and allowed us to observe two phosphate ions (Figure 6A) of the crystallant bound to the surface of this RNA binding enzyme.

Lysozyme crystals were analyzed at room temperature using the X-ray lab source from the INSTRUCT-FRISBI platform at the IGBMC (Illkirch, France). A fast data collection was applied using

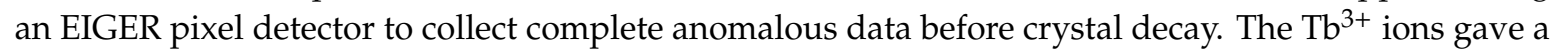
strong anomalous signal at $1.5418 \AA$ ( $\mathrm{f}^{\prime \prime}$ of 9 electrons at the $\mathrm{Cu} \mathrm{K} \alpha$ wavelength). The dataset collected at $1.51 \AA$ resolution (Table 1) allowed the identification of three anomalous peaks corresponding to $\mathrm{Tb}^{3+}$ sites, a major site with an occupancy of $\mathrm{q}=0.8$ for which the ligand could be built, and a minor site with two alternate positions (Figure 6C). These sites are consistent with those described in PDBid 6F2I determined with an HEWL crystal grown in $0.8 \mathrm{M} \mathrm{NaCl}$ and $100 \mathrm{mM} \mathrm{Tb}-\mathrm{Xo}_{0}$ [20]. The two $\mathrm{Tb}^{3+}$ positions corresponding to the minor site have a low occupancy $(\mathrm{q}=0.2$ and 0.4$)$ and no visible ligand, which is also consistent with the low concentration of $\mathrm{Tb}-\mathrm{Xo}_{\mathrm{o}}(10 \mathrm{mM})$ providing a ratio of two $\mathrm{Tb}-\mathrm{Xo} 4$ complexes for one lysozyme molecule. This concentration seems to be sufficient to trigger the 
association of lysozyme particles, nucleation and subsequent crystal growth, but not to guarantee a full occupancy in the crystal lattice.

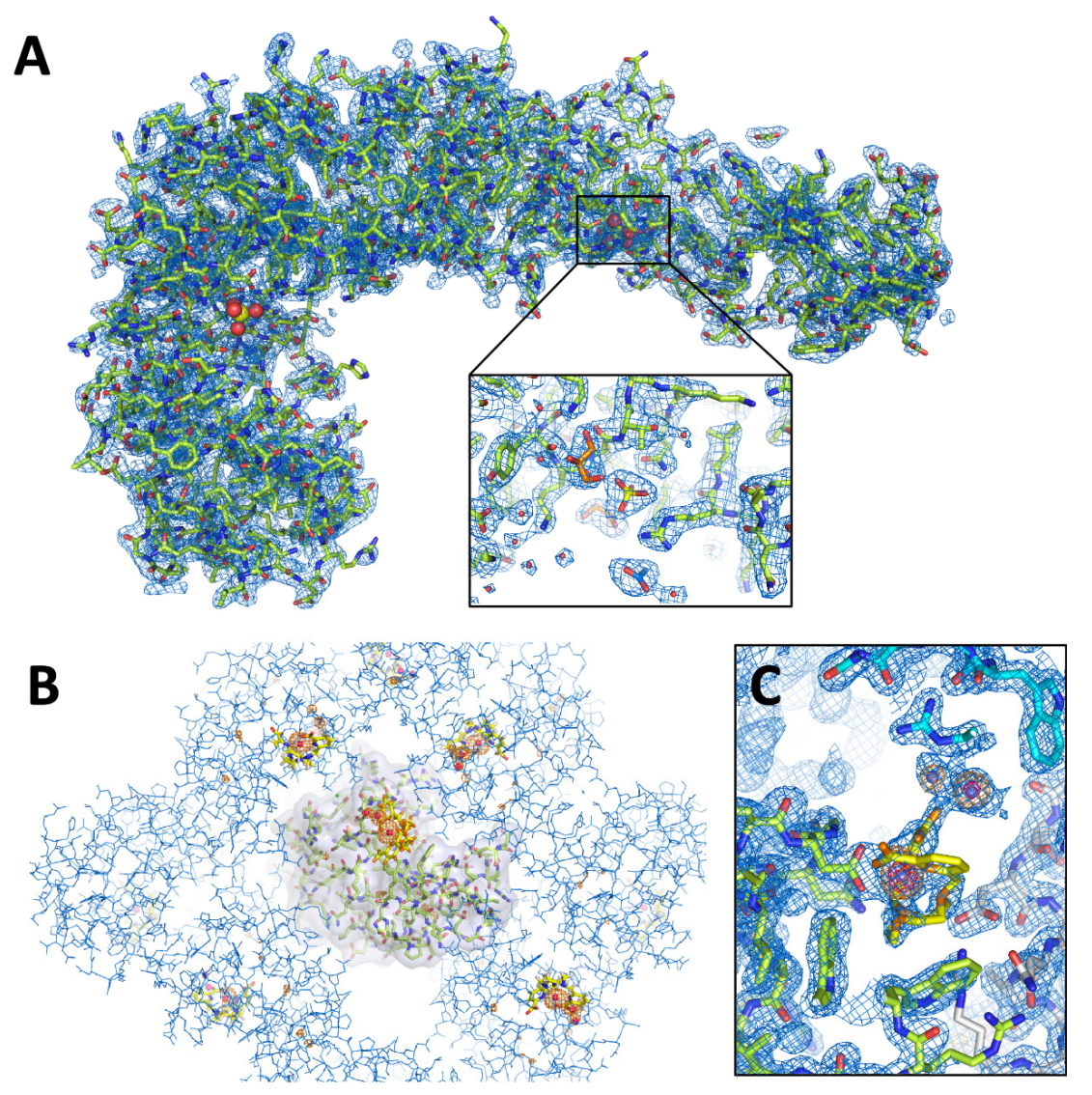

Figure 6. 3D structures of PhaCCA and HEWL crystallized in the XtalController. (A) Overall structure of PhaCCA and close-up on a phosphate, an acetate and two glycerol molecules bound to the enzyme surface. The blue $2 m F o-D F c$ map is contoured at $1.2 \sigma$. (B) View of tetragonal crystal packing of HEWL seen down the four-fold axis. One lysozyme monomer is represented with a $\mathrm{Tb}-\mathrm{Xo}_{\mathrm{o}}$ complex bound to its surface. Symmetry related monomers are depicted in line mode. The anomalous difference map shown in orange is contoured at $5 \sigma$ and indicates the presence of $\mathrm{Tb}^{3+}$ ions (red spheres) as well as sulfur atoms. (C) Zoom on the Tb-Xo4 binding sites. The side chain of Asp101 directly coordinates the $\mathrm{Tb}^{3+}$ of the major site for which the organic ligand (in yellow) is partially visible in the blue $2 m F o-D F c$ map contoured at $1 \sigma$. The anomalous difference map depicted in orange and red is contoured at 5 and $20 \sigma$, respectively, and highlights the strong anomalous signal of $\mathrm{Tb}^{3+}$ ions. Two alternate positions are observed but their ligand is not visible due to low occupancy. The two adjacent $\mathrm{Tb}-\mathrm{Xo}_{0}$ sites constitute a bridge (molecular glue) between the green and blue lysozyme monomers.

\section{Conclusions}

In the perspective of emerging time-resolved studies of enzyme:substrate systems using SFX and XFEL facilities, it becomes increasingly important to gain more control over sample production, quality and reproducibility. These two examples of enzyme crystallization highlight the usefulness of the XtalController to monitor the evolution of crystallization assays and to act on the process. Beyond helping define and optimize crystallization conditions, the XtalController and its integrated DLS module may also be an ideal tool to:

- Explore phase diagrams of biomolecules with a direct feedback on nucleation events;

- Study the stability of biomolecules in solution with respect to various parameters such as temperature, $\mathrm{pH}$, ligands, etc.; 
- Determine the optimum conditions for introducing a cryoprotectant;

- Ensure the reproducibility of crystals in the context of structural biology investigations, rational drug design, and fragment-based screening.

- Produce calibrated nanocrystals on demand (difficult to monitor and control otherwise) for diffraction analyses using X-ray free electron lasers and $\mu \mathrm{ED}$, or, conversely, to promote the selective growth of large crystals for neutron diffraction.

More generally, this type of versatile instruments provides a more rational approach to crystallization and a great alternative to extensive blind screening. We do believe that this technology has a bright future.

Author Contributions: Formal analysis, R.D.W., K.R., E.G., P.B., B.L. and C.S.; funding acquisition, M.M., O.M., E.G. and C.S.; investigation, R.D.W., K.R., S.E., A.G.M., O.H., O.M., E.G., P.B., B.L. and C.S.; resources, F.R. and O.M.; supervision, M.M., O.M., E.G. and C.S.; writing—original draft, C.S.; writing—review and editing, R.D.W., K.R., S.E., A.G.M., O.H., H.B., M.M., F.R., O.M., E.G., P.B., B.L. and C.S. All authors have read and agreed to the published version of the manuscript.

Funding: This work was supported by the French Centre National de la Recherche Scientifique (CNRS), the University of Strasbourg, the project Ln23 (ANR-13-BS07-0007-01), the LabEx consortia "NetRNA" (ANR-10-LABX-0036_NETRNA) and "INRT" (ANR-10-LABX-0036_INRT), a French State fund managed by the Agence National de la Recherche under the frame program Investissements d'Avenir ANR-10-IDEX-0002-02, a PhD funding to R.dW from the Excellence initiative (IdEx) of the University of Strasbourg, a PhD funding to K.R from the French-German University (UFA/DFH), the Deutsche Forschungsgemeinschaft (grant no. Mo 634/10-1). The authors benefitted from the PROCOPE Hubert Curien cooperation program (French Ministry of Foreign Affair and Deutscher Akademischer Austauschdienst). The XtalController was acquired with the financial support of the LabEx consortium “MitoCross” (ANR-11-LABX-0057_MITOCROSS).

Acknowledgments: The authors thank the team of FIP beamline at the European Synchrotron Radiation Facility (ESRF, Grenoble, France) for beamtime allocation; Alexandra Bluhm and Léna Coudray for their contribution to crystallization experiments and structure refinement; Robin Schubert and Christian Betzel (University of Hamburg), as well as Karsten Dierks, Arne Mayer and Annette Eckhardt (Xtal-Concepts GmbH, Hamburg) for fruitful discussions and exchanges about the XtalController. The authors acknowledge the support and use of the resources of the French Infrastructure for Integrated Structural Biology (FRISBI, ANR-10-INBS-05) and of Instruct-ERIC.

Conflicts of Interest: The authors declare no conflict of interest.

\section{References}

1. Giegé, R.; Sauter, C. Biocrystallography: Past, present, future. HFSP J. 2010, 4, 109. [CrossRef] [PubMed]

2. Chapman, H.N.; Fromme, P.; Barty, A.; White, T.A.; Kirian, R.A.; Aquila, A.; Hunter, M.S.; Schulz, J.; DePonte, D.P.; Weierstall, U.; et al. Femtosecond X-ray protein nanocrystallography. Nature 2011, 470, 73-77. [CrossRef] [PubMed]

3. Spence, J.C.H.; Chapman, H.N. The birth of a new field. Philos. T. Roy. Soc. B 2014, 369, 20130309. [CrossRef] [PubMed]

4. Johansson, L.C.; Stauch, B.; Ishchenko, A.; Cherezov, V. A Bright Future for Serial Femtosecond Crystallography with XFELs. Trends Biochem. Sci. 2017, 42, 749-762. [CrossRef]

5. Shi, D.; Nannenga, B.L.; Iadanza, M.G.; Gonen, T. Three-dimensional electron crystallography of protein microcrystals. Elife 2013, 2, e01345. [CrossRef]

6. Nannenga, B.L.; Shi, D.; Leslie, A.G.W.; Gonen, T. High-resolution structure determination by continuous-rotation data collection in MicroED. Nat. Methods 2014, 11, 927-930. [CrossRef]

7. Clabbers, M.T.B.; van Genderen, E.; Wan, W.; Wiegers, E.L.; Gruene, T.; Abrahams, J.P. Protein structure determination by electron diffraction using a single three-dimensional nanocrystal. Acta Cryst. D 2017, 73, 738-748. [CrossRef]

8. Nederlof, I.; Li, Y.W.; van Heel, M.; Abrahams, J.P. Imaging protein three-dimensional nanocrystals with cryo-EM. Acta. Crystallogr. D. Biol Crystallogr. 2013, 69, 852-859. [CrossRef]

9. Sauter, C.; Lorber, B.; McPherson, A. Crystallization-General Methods. In International Tables of Crystallography, Vol. F, Crystallography of Biological Macromolecules, 2nd ed.; Arnold, E., Himmel, D.M., Rossmann, M.G., Eds.; John Wiley and Sons: Chichester, UK, 2012; pp. 99-120. 
10. Garcia-Caballero, A.; Gavira, J.A.; Pineda-Molina, E.; Chayen, N.E.; Govada, L.; Khurshid, S.; Saridakis, E.; Boudjemline, A.; Swann, M.J.; Shaw Stewart, P.; et al. Optimization of Protein Crystallization: The OptiCryst Project. Cryst. Growth Des. 2011, 11, 2112-2121. [CrossRef]

11. Meyer, A.; Dierks, K.; Hilterhaus, D.; Klupsch, T.; Mühlig, P.; Kleesiek, J.; Schöpflin, R.; Einspahr, H.; Hilgenfeld, R.; Betzel, C. Single-drop optimization of protein crystallization. Acta Crystallog. F 2012, 68, 994-998. [CrossRef]

12. Mikol, V.; Hirsch, E.; Giegé, R. Diagnostic of precipitant for biomacromolecule crystallization by quasi-elastic light-scattering. J. Mol. Biol. 1990, 213, 187-195. [CrossRef]

13. Kadima, W.; McPherson, A.; Dunn, M.F.; Jurnak, F.A. Characterization of precrystallization aggregation of canavalin by dynamic light scattering. Biophys. J. 1990, 57, 125-132. [CrossRef]

14. George, A.; Wilson, W.W. Predicting protein crystallization from a dilute solution property. Acta Cryst. D Biol Cryst. 1994, 50, 361-365. [CrossRef]

15. Zulauf, M.; D'Arcy, A. Light scattering of proteins as a criterion for crystallization. J. Cryst. Growth. 1992, 122, 102-106. [CrossRef]

16. Dierks, K.; Meyer, A.; Einspahr, H.; Betzel, C. Dynamic Light Scattering in Protein Crystallization Droplets: Adaptations for Analysis and Optimization of Crystallization Processes. Cryst. Growth Des. 2008, 8, 1628-1634. [CrossRef]

17. Schubert, R.; Meyer, A.; Baitan, D.; Dierks, K.; Perbandt, M.; Betzel, C. Real-Time Observation of Protein Dense Liquid Cluster Evolution during Nucleation in Protein Crystallization. Cryst. Growth Des. 2017, 17, 954-958. [CrossRef]

18. Baitan, D.; Schubert, R.; Meyer, A.; Dierks, K.; Perbandt, M.; Betzel, C. Growing Protein Crystals with Distinct Dimensions using Automated Crystallization Coupled with in Situ Dynamic Light Scattering. JoVE. 2018, e57070. [CrossRef]

19. Engilberge, S.; Riobé, F.; Di Pietro, S.; Lassalle, L.; Coquelle, N.; Arnaud, C.-A.; Pitrat, D.; Mulatier, J.-C.; Madern, D.; Breyton, C.; et al. Crystallophore: A versatile lanthanide complex for protein crystallography combining nucleating effects, phasing properties, and luminescence. Chem. Sci. 2017, 8, 5909-5917. [CrossRef]

20. Engilberge, S.; Riobé, F.; Wagner, T.; Pietro, S.D.; Breyton, C.; Franzetti, B.; Shima, S.; Girard, E.; Dumont, E.; Maury, O. Unveiling the Binding Modes of the Crystallophore, a Terbium-based Nucleating and Phasing Molecular Agent for Protein Crystallography. Chemistry 2018, 24, 9739-9746. [CrossRef]

21. De Wijn, R.; Hennig, O.; Ernst, F.G.M.; Lorber, B.; Betat, H.; Mörl, M.; Sauter, C. Combining crystallogenesis methods to produce diffraction-quality crystals of a psychrophilic tRNA-maturation enzyme. Acta Crystallog. F 2018, 74, 747-753. [CrossRef]

22. Kabsch, W. XDS. Acta Crystallogr. D. Biol. Crystallogr. 2010, 66, 125-132. [CrossRef] [PubMed]

23. Adams, P.D.; Afonine, P.V.; Bunkóczi, G.; Chen, V.B.; Davis, I.W.; Echols, N.; Headd, J.J.; Hung, L.-W.; Kapral, G.J.; Grosse-Kunstleve, R.W.; et al. PHENIX: A comprehensive Python-based system for macromolecular structure solution. Acta Crystallogr. D. Biol. Cryst. 2010, 66, 213-221. [CrossRef] [PubMed]

24. Emsley, P.; Cowtan, K. Coot: Model-building tools for molecular graphics. Acta Crystallogr. D. Biol. Cryst. 2004, 60, 2126-2132. [CrossRef] [PubMed]

25. De Wijn, R.; Hennig, O.; Roche, J.; Engilberge, S.; Rollet, K.; Fernandez-Millan, P.; Brillet, K.; Betat, H.; Mörl, M.; Roussel, A.; et al. A simple and versatile microfluidic device for efficient biomacromolecule crystallization and structural analysis by serial crystallography. IUCrJ 2019, 6, 454-464. [CrossRef]

26. Junius, N.; Oksanen, E.; Terrien, M.; Berzin, C.; Ferrer, J.-L.; Budayova-Spano, M. A crystallization apparatus for temperature-controlled flow-cell dialysis with real-time visualization. J. Appl. Cryst. 2016, 49, 806-813. [CrossRef]

27. Engilberge, S.; Wagner, T.; Santoni, G.; Breyton, C.; Shima, S.; Franzetti, B.; Riobé, F.; Maury, O.; Girard, E. Protein crystal structure determination with the crystallophore, a nucleating and phasing agent. J. Appl. Cryst. 2019, 52, 722-731. [CrossRef]

(C) 2020 by the authors. Licensee MDPI, Basel, Switzerland. This article is an open access article distributed under the terms and conditions of the Creative Commons Attribution (CC BY) license (http://creativecommons.org/licenses/by/4.0/). 